\title{
Pulsations in white dwarf stars
}

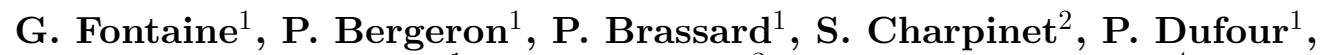 \\ N. Giammichele ${ }^{1}$, S. K. Randall ${ }^{3}$, and V. Van Grootel ${ }^{4}$ \\ ${ }^{1}$ Département de Physique, Université de Montréal, \\ Montréal, Québec, Canada H3C 3J7 \\ email: fontaine@astro.umontreal.ca \\ ${ }^{2}$ CNRS, Université de Toulouse, UPS-OMP, IRAP, \\ 14 av. E. Belin, 31400 Toulouse, France \\ ${ }^{3}$ European Southern Observatory, Karl-Schwarzschild-Str. 2 \\ 85748 Garching bei München, Germany \\ ${ }^{4}$ Institut d'Astrophysique et de Géophysique de l'Université de Liège \\ Allée du 6 Août 17, B-4000 Liège, Belgique
}

\begin{abstract}
We first present a brief description of the six distinct families of pulsating white dwarfs that are now known. These are all opacity-driven pulsators showing low- to mid-order, low-degree gravity modes. We then discuss some recent highlights that have come up in the field of white dwarf asteroseismology.
\end{abstract}

Keywords. stars: oscillations, white dwarfs

\section{The families of pulsating white dwarfs}

A general view of the distribution of known pulsating stars lying at the bottom of the spectroscopic H-R diagram is provided by Fig. 1. Note that only those pulsators with reliable estimates of their atmospheric parameters have been plotted. There exist many more pulsating stars of the kinds, particularly of the ZZ Cet and V777 Her type, but those have yet to be well characterized spectroscopically speaking. One can identify at least six different categories of pulsating white dwarfs, and three different types of pulsating hot subdwarfs. The properties of the latter are reviewed by S. Randall et al. in these proceedings, and will no longer be referred to in this article.

The ZZ Cet pulsators are H-atmosphere (or DA) white dwarfs found in a very narrow instability strip in the lower right corner of the H-R diagram as indicated in Fig. 1. The DA white dwarfs themselves constitute some $80 \%$ of post-AGB stars and, hence, form the majority of white dwarfs. A representative evolutionary track for a $0.593 M_{\odot}$ DA star connects the post-AGB domain with the ZZ Cet instability strip in the figure. The pulsations detected in ZZ Cet stars correspond to low- to mid-order, low-degree g modes. The ZZ Cet stars are opacity-driven pulsators. The exact excitation mechanism is sometimes referred to as convective driving, but the ultimate culprit is the recombination of $\mathrm{H}$ in the envelope as a cooling DA white dwarf enters and then transits across a narrow strip.

A closely related family is that of the V777 Her stars which involves, this time, Heatmosphere (or DB) white dwarfs. The latter are the cool descendants of the other $20 \%$ of post-AGB objects. That smaller fraction corresponds to stars that experience a very late He flash in their evolution, which causes a violent mixing episode that destroys the remaining $\mathrm{H}$ in these objects. Thus, about $20 \%$ of all white dwarfs are born devoid of hydrogen. The DB stars descend from those H-deficient objects (a representative case is illustrated by the dashed evolutionary track) and constitute a spectral type based 


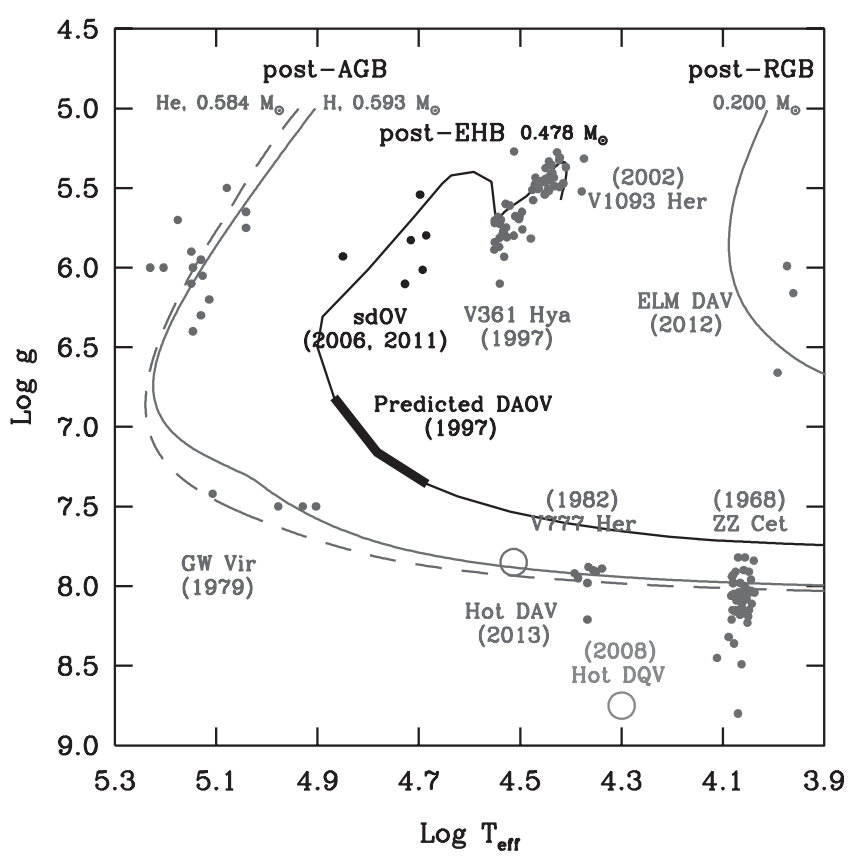

Figure 1. Regions of the $\log g$ - $\log T_{\text {eff }}$ plane where the several families of compact pulsators are found. The year of the discovery of the prototype of each class is indicated. Five of the distinct classes are identified by their official IAU names, while the others, more recently discovered, have yet to be given IAU variable star names. To guide the eye, four representative evolutionary tracks have been plotted. Further details are provided in the text.

on He I lines visibility, concentrated between about $30000 \mathrm{~K}$ and $12000 \mathrm{~K}$ in effective temperature. The pulsations detected in V777 Her DB white dwarfs are of the same nature as those found in ZZ Cet stars. Convective driving is again at work, this time associated with the recombination of He in the envelope. The V777 Her instability strip is found at higher effective temperatures than the ZZ Cet strip because the ionization potentials of He I and He II are larger than that of H I.

The hottest pulsating stars known are the GW Vir white dwarfs found on the left side of Fig. 1. They span a huge domain in effective temperature and surface gravity. These pulsators are white dwarfs that belong to the PG1159 spectral type, characterized by mixed He-C-O atmospheres. The PG1159 white dwarfs are the immediate, very hot descendants of that fraction of $20 \%$ of post-AGB stars that underwent a late He flash. Their atmospheres/envelopes still reflect a composition (a mixture of $\mathrm{He}, \mathrm{C}$, and $\mathrm{O}$ in roughly comparable proportions, but which varies from star to star) that resulted from that mixing event. Ultimately, gravitational settling of $\mathrm{C}$ and $\mathrm{O}$ produces almost pure He atmopsheres, and PG1159 stars evolve into cooler white dwarfs of the DO and DB spectral types. The pulsations observed in GW Vir stars are again low- to mid-order, lowdegree $g$ modes. The excitation mechanism is now a classical $\kappa$-mechanism (convection has little impact in these very hot stars) associated with the presence of opaque high ions of $\mathrm{C}$ and $\mathrm{O}$ in the envelope.

An extremely rare type of white dwarfs, the so-called Hot DQ stars, has been discovered relatively recently by Dufour et al. (2007). These are C-atmosphere white dwarfs that bunch around $20000 \mathrm{~K}$ in effective temperature and have relatively high surface gravities by white dwarf standards (see Fig. 1). Their exact origin is uncertain and they are still being characterized. They appear to be highly magnetic (with field strengths exceeding 
1 MGauss) and half of them have been found to pulsate. The pulsations observed would correspond to mid- to low-order, low-degree g modes. Convective driving associated with the recombination of $\mathrm{C}$ in the envelope is the mechanism responsible for the excitation of pulsation modes in these Hot DQV variables.

Gravity-mode pulsations have been detected recently in three extremely low-mass (ELM) DA white dwarfs (Hermes et al. 2012, 2013a). The locations of these variable stars are indicated in Fig. 1 , and a representative $0.2 M_{\odot}$ evolutionary track is also plotted. The ELM white dwarfs are relatively rare objects produced by binary (common envelope) evolution. They are post-RGB remnants and are constituted of a He core surrounded by a relatively thick $\mathrm{H}$ envelope. The pulsations detected correspond to lowto mid-order, low-degree g modes. Given their much lower surface gravities (and considerably reduced average densities), the periods involved are much longer (thousands of seconds versus hundreds of seconds) compared to other pulsating white dwarfs.

An even more recent development has been the report of Kurtz et al. (2013) concerning the probable discovery of yet another class of pulsating white dwarfs. The newlyuncovered variable stars are DA white dwarfs that cluster around $30000 \mathrm{~K}$ in effective temperature (see Fig. 1), and should be referred to as Hot DAV white dwarfs. The tentative explanation is that these stars have very thin $\mathrm{H}$ outer layers sitting on top of a He mantle in which the driving/damping region resides. In effect, these objects are DBs "disguised" as DAs. Low-order, low-degree g-mode pulsations would be driven by the same opacity mechanism that operates in V777 Her stars.

Finally, we recall a prediction made by Charpinet et al. (1997) to the effect that low-mass, post-EHB DAO white dwarfs should show very low-order, low-degree g-mode pulsations driven by the $\epsilon$-mechanism associated with $\mathrm{H}$-shell burning at the base of the $\mathrm{H}$ envelope in these stars. The heavy curve segment along the illustrated post-EHB track in Fig. 1 identifies the domain where $\epsilon$-driving should be in action. It should be pointed out here that post-EHB evolution contributes at most $2 \%$ of the total white dwarf population. In addition, the evolutionary phase indicated by the heavy curve segment is relatively rapid, meaning that these hot, low-mass DA white dwarfs are quite rare. And indeed, a real pulsator of the kind has yet to be found, but the prediction has been made.

\section{Recent highlights in white dwarf seismology}

In the rest of this contribution, we focus on some recent highlights of interest in the field of white dwarf seismology. The reader will find an exhaustive review of the progress made in this field up to 2008 in Fontaine \& Brassard (2008).

\subsection{An enlightening discussion}

Saio (2013) has recently presented a brief but, in our view, particularly interesting discussion on the driving mechanisms in pulsating white dwarfs. For those interested in a deeper physical understanding of these mechanisms as well as a first acquaintance with the effects of large magnetic fields in pulsating white dwarfs, this is highly recommended reading.

\subsection{The total angular momentum of white dwarfs}

Seismology has been successfully used by Charpinet et al. (2009) to map the internal rotation profile of PG 1159-035, the prototype of the PG1159 spectral type for white dwarfs, and also the prototype of the GW Vir class of pulsating white dwarfs. This is depicted in Fig. 2, which shows that some $99 \%$ of the mass of the star has been covered, thus allowing one to compute essentially the total angular momentum and total 


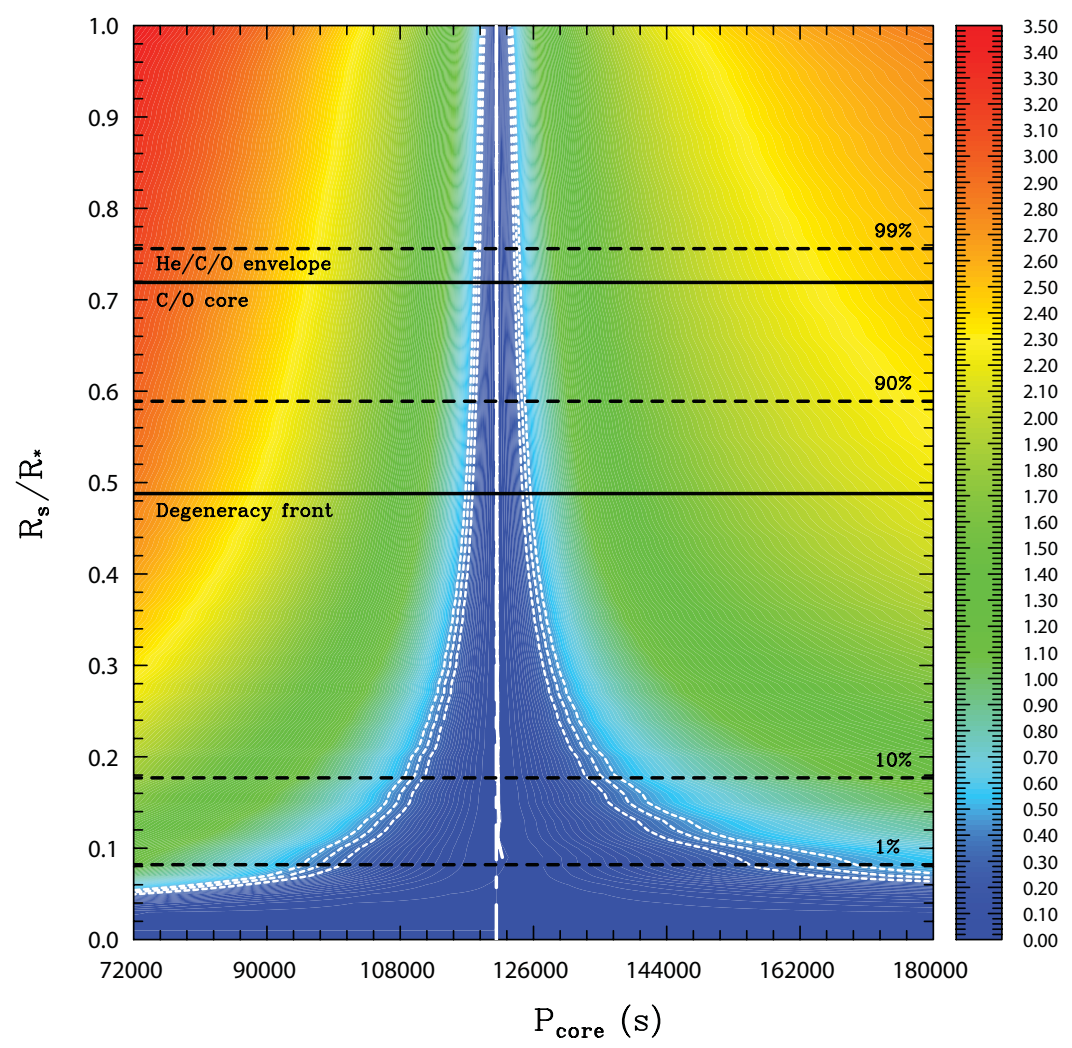

Figure 2. Internal rotation profile of the GW Vir star PG 1159-035 illustrated as depth (on a radius scale) versus the local rotation period. The dashed horizontal lines labelled $99 \%, 90 \%$, $10 \%$, and $1 \%$ correspond to the levels in the seismic model below which $99 \%, 90 \%, 10 \%$, and $1 \%$ of the total mass is concentrated. The sounding method loses its sensitivity over the inner $\sim 10 \%$ of the radius (as indicated by the diverging confidence curves depicting the 1,2 , and 3 $\sigma$ levels), but this inner region contains only about $1 \%$ of the total mass. This implies that the rotation profile is secure over some $99 \%$ of the mass of the model.

rotation energy of that object. The rotation profile in PG 1159-035 corresponds to solid body rotation. Much more importantly, Fig. 2 reveals that PG 1159-035 rotates in bulk extremely slowly by white dwarf standards, with $P_{\text {rot }}=33.67 \pm 0.24 \mathrm{~h}$. While it has been known for several decades that isolated white dwarfs are slow rotators at their surfaces (through spectroscopic or polarization measurements), one could never exclude the possibility that their cores could spin quite rapidly, thus "hiding" most of their angular momentum.

We have recently carried out similar asteroseismic analyses for the three additional GW Vir stars with rotation data available in the literature. The results are qualitatively the same as that obtained for PG 1159-035, i.e., these objects all rotate solidly and extremely slowly. In fact the ratio of the total rotation energy to the thermal energy in these objects varies from $10^{-6}$ in our fastest rotator to $10^{-8}$ in our slowest one, meaning that, for all practical purposes, these stars have lost all of their angular momentum. Given that these four objects are representative of single star evolution, this finding has very important implications for theories of angular momentum transfer between the radiative core and the convective envelope in red giant phases leading to the final white dwarf phase in stellar evolution. 


\subsection{An updated view of the ZZ Cet instability strip}

Considerable efforts have been invested at Université de Montréal over the last two decades to provide a reliable and homogeneous description of the empirical ZZ Cet instability strip, starting with the pioneering efforts of Bergeron et al. (1995). Emphasis has been put on gathering high $\mathrm{S} / \mathrm{N}$ spectroscopic data using the same experimental setup, making sure that the exposure times exceed the dominant periods (in order to obtain meaningful time-averaged spectra), and deriving atmospheric parameters in a strict homogeneous way with the help of state-of-the-art model atmospheres. Gianninas et al. (2005) demonstrated that without using these stringent criteria, the view of the empirical ZZ Cet strip remains blurred, in particular when using the low $\mathrm{S} / \mathrm{N}$ spectra coming out of the SDSS survey. In contrast, Fig. 3 (built by adhering stricly to the above approach) provides the best available view of the empirical ZZ Cet instability domain in the $\log g$ - $T_{\text {eff }}$ diagram.

Considerable progress has also been made in recent years on the theory front, thanks mainly to the introduction of time-dependent convection (TDC) into nonadiabatic calculations as applied to ZZ Cet pulsators at Université de Liège (Van Grootel et al. 2012). Assuming that convection can be described by the so-called ML2 $/ \alpha=1.0$ version of the mixing-length theory, convective driving described with TDC leads to a theoretical blue edge given by the solid curve on the left in Fig. 3. However, TDC still fails at explaining the empirical red edge, so Van Grootel et al. (2013) have proposed a semi-analytic criterion based on the comparison between the thermal timescale at the base of the convection zone (where the driving region is located in ZZ Cet models) and the cutoff g-mode period (due to energy leakage through the atmosphere). This criterion leads to a theoretical red edge (for dipole modes) given by the solid curve on the right in Fig. 3. Given that the red edge location is sensitive to the assumed convective efficiency used in the equilibrium model, and given that it is obtained independently of the TDC nonadiabatic calculations carried out for obtaining the blue edge, Fig. 3 provides a most satisfying view of the ZZ Cet instability strip. This is the first time that such a good agreement has been obtained between theory and observations. Furthermore, the fact that the three recentlydiscovered pulsating ELM DA white dwarfs fall into the extension of the theoretical strip into the low-gravity regime is a convincing proof that these are genuine ZZ Cet stars, driven by the same mechanism, but with very low masses. Further details are provided in Van Grootel et al. (2013).

\subsection{Discovery of an ultramassive ZZ Cet pulsator}

Hermes et al. (2013b) have reported the discovery of $g$-mode pulsations in GD 518, a DA white dwarf (a ZZ Cet star) massive enough to be partly solidified in its core. This is the second pulsating star of the kind, the first one being BPM 37093 discussed, for instance, by Metcalfe et al. (2004) and Brassard \& Fontaine (2005). GD 518 is the most massive pulsating white dwarf ever found and it is likely to have a core made of neonoxygen instead of the more standard carbon-oxygen composition. Asteroseismology may yet reveal its true nature. In Fig. 3, GD 518 appears as the lowest (highest gravity) data point, sitting at the red edge of the instability strip, a position consistent with the observed periods since there exist period-effective temperature relationships sensitive on the mass for ZZ Cet stars (see, e.g., Fig. 24 of Brassard \& Fontaine 2008). In comparison, BPM 37093 sits just above and slightly to the right of GD 518 in Fig. 3.

\subsection{Finally making sense of the $G W$ Vir instability domain}

The puzzle of the GW Vir instability strip has finally been resolved by Quirion, Fontaine, \& Brassard (2012) who presented a first coherent picture of the phenomenon by invoking 


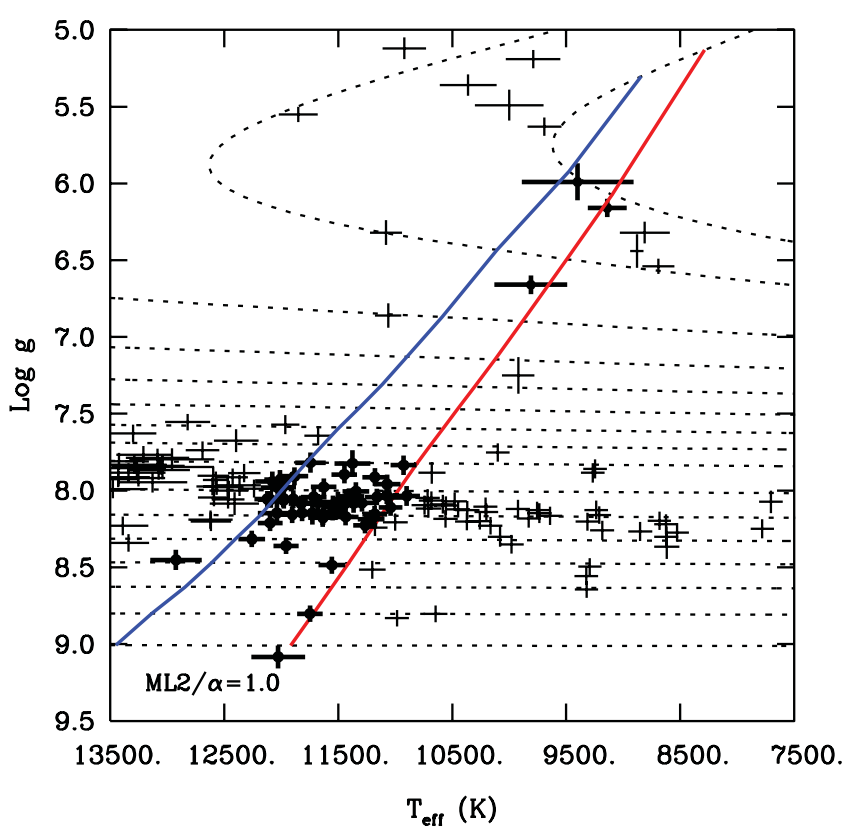

Figure 3. Comparison of the empirical with the theoretical instability domain in the $\log g-T_{\text {eff }}$ diagram for pulsating DA white dwarfs. Only the stars with reliable and homogeneous determinations of their spectroscopic parameters are plotted here. The heavy symbols indicate the pulsators, while the light crosses correspond to stars that have been monitored for variability but found not to vary. The theoretical boundaries of the strip have been obtained from models computed with the so-called ML2/ $\alpha=1.0$ version of the mixing-length theory. With this convective efficiency, the predicted $l=1$ blue edge is given by the solid curve on the left as obtained from detailed nonadiabatic calculations using TDC. For its part, the predicted $l=1$ red edge is given by the solid curve on the right and was obtained from an independent semi-analytic argument involving the local thermal timescale in the driving region and the cutoff period for dipole g modes.

the competing actions of a stellar wind and of gravitational settling. As indicated above, PG1159 stars are the immediate descendants of "born-again" post-AGB stars that have undergone a late He flash. Their atmosphere/envelope compositions are mixtures of He, $\mathrm{C}$, and $\mathrm{O}$ in very roughly comparable proportions, but varying from one object to another. Those PG1159 stars with compositions dominated by $\mathrm{C}$ and $\mathrm{O}$ over He (about half of them) pulsate as GW Vir variables as they contain enough opaque ions of $\mathrm{C}$ and $\mathrm{O}$ in the driving region (a pure $\kappa$-mechanism is at work here). The problem has been how to explain and maintain such mixed compositions in high-gravity objects, and how to account for the empirical blue and red edges of the instability domain.

Figure 4 illustrates what happens if one artificially maintains a representative GW Vir atmosphere/envelope composition in an evolving model. As indicated by the filled circles, the instability domain then extends all the way down to about $\sim 30000 \mathrm{~K}$ in effective temperature. However, mixed-atmosphere white dwarfs of the sort do not exist below the GW Vir empirical red edge which is located between $\sim 70000 \mathrm{~K}$ and $\sim 80000 \mathrm{~K}$, depending on the surface gravity (mass). Hence, the initial composition cannot be maintained over long evolutionary timescales and the most obvious cause for this state of affair is the action of diffusion, which cannot be turned off. And the process is particularly efficient in very high-gravity stars such as white dwarfs. 


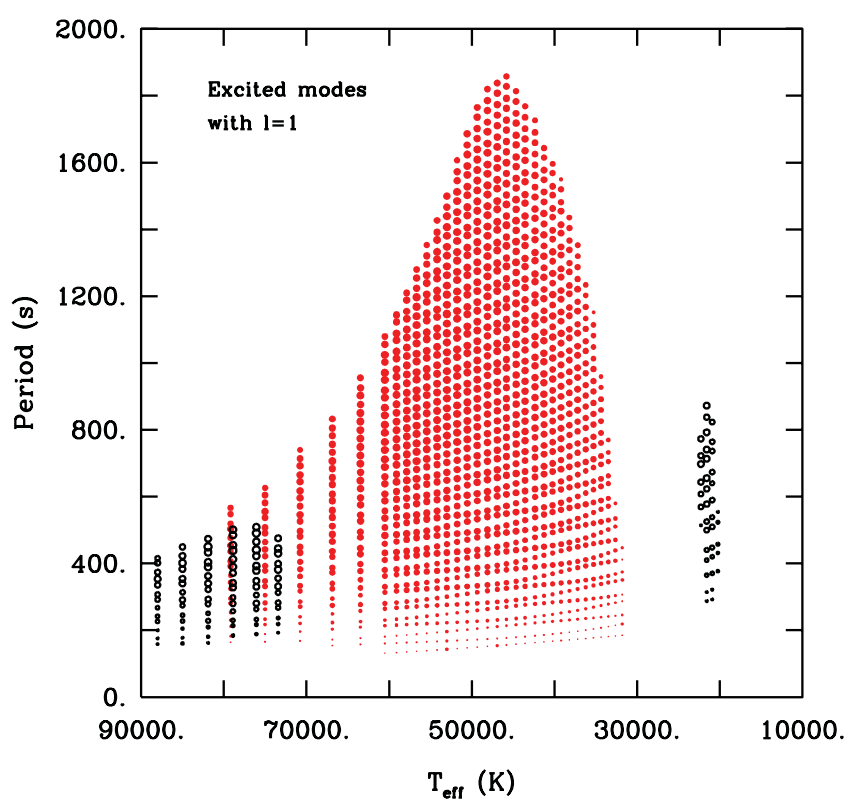

Figure 4. Period spectra of excited dipole modes in two post-PG1159 evolutionary models. The filled circles refer to a model with a fixed envelope chemical composition specified by $X(\mathrm{He})=$ $0.38, X(\mathrm{C})=0.40, X(\mathrm{O})=0.20$, and $Z=0.02$. The open circles refer to a similar $0.6 M_{\odot}$ model defined by the same initial conditions but for which diffusion and mass loss have been turned on in the calculations. In the latter sequence, a GW Vir red edge is naturally found around $T_{\text {eff }}=$ $75000 \mathrm{~K}$, and a V777 Her instability phase is also recovered near the end of the evolution. Each circle gives the period of a mode, and its size represents a logarithmic measure of the modulus of the imaginary part $\sigma_{\mathrm{I}}$ of the complex eigenfrequency. The bigger the circle, the more unstable the mode.

On the other hand, if left unimpeded, diffusion will transform the atmosphere of a PG1159 star into a pure He atmosphere almost instantly, and will lead to the settling of the opaque $\mathrm{C}$ and $\mathrm{O}$ ions out of the driving region in less than a few hundred years, thus stopping pulsational driving. In other words, white dwarfs of spectral type PG1159 (and their associated GW Vir pulsators) would not exist in that scenario. Thus, clearly, some mechanism must slow down the effects of gravitational settling over the timespan of the PG1159 evolutionary phase, which lasts about $2 \times 10^{6} \mathrm{yr}$, typically. Note that radiative levitation can only maintain small amounts of $\mathrm{C}$ and $\mathrm{O}$ in the atmosphere/envelope of a PG1159 star, certainly not enough to feed a $\kappa$ mechanism. Instead, Quirion et al. (2012) have proposed the competing effect of a stellar wind, which appears a natural candidate in such very hot stars (and indeed, direct evidence for the presence of a wind exists for some of the most luminous PG1159 stars). Details are provided in the paper.

An animation summarizing these considerations has been presented at the conference. The interested reader may look up that animation in the .ppt presentation of G.F. available on the official web site of the meeting: http://iaus301.astro.uni.wroc.pl.

\section{References}

Bergeron, P., Wesemael, F., Lamontagne, R., Fontaine, G., Saffer, R. A., \& Allard, N. F. 1995, ApJ, 449, 258

Brassard, P. \& Fontaine, G. 2005, ApJ, 622, 572

Charpinet, S., Fontaine, G., \& Brassard, P. 1997, ApJ, 489, L149 
Charpinet, S., Fontaine, G., \& Brassard, P. 2009, Nature, 561, 501

Dufour, P., Liebert, J., Fontaine, G., \& Behara, N. 2007, Nature, 450, 522

Fontaine, G. \& Brassard, P. 2008, PASP, 120, 1043

Gianninas, A., Bergeron, P., \& Fontaine, G. 2005, ApJ, 631, 1100

Hermes, J. J., Montgomery, M. H., Winget, D. E., Brown, W. R., Kilic, M., \& Kenyon, S. C. 2012, ApJ, 750, L28

Hermes, J. J., Montgomery, M. H., Winget, D. E., et al. 2013a, ApJ, 765, 102

Hermes, J. J., Kepler, S. O., Castanheira, B., et al. 2013b, ApJ, 771, L2

Kurtz, D. W., Shibahashi, H., Dhillon, V. S., et al. 2013, MNRAS, 432, 1632

Metcalfe, T. S., Montgomery, M. H., \& Kanaan, A. 2004, ApJ, 605, L133

Quirion, P.-O., Fontaine, G., \& Brassard, P. 2012, ApJ, 755, 128

Saio, H. 2013, in: J. Montalbán, A. Noels, \& V. Van Grootel (eds.), Ageing Low Mass Stars: From Red Giants to White Dwarfs, EPJ Web of Conferences, 43, 05005

Van Grootel, V., Dupret, M.-A., Fontaine, G., Brassard, P., Grigahcène, A., \& Quirion, P.-O. 2012, A\& $\mathcal{E}, 539, \mathrm{~A} 87$

Van Grootel, V., Fontaine, G., Brassard, P., \& Dupret, M.-A. 2013, ApJ, 762, 57 